\title{
Harmonic Analysis \\ on Quantum Complex Hyperbolic Spaces
}

\author{
Olga BERSHTEIN and Yevgen KOLISNYK
}

Institute for Low Temperature Physics and Engineering, 47 Lenin Ave., 61103, Kharkov, Ukraine

E-mail: bershtein@ilt.kharkov.ua, evgen.kolesnik@gmail.com

Received April 30, 2011, in final form August 10, 2011; Published online August 18, 2011

http://dx.doi.org/10.3842/SIGMA.2011.078

\begin{abstract}
In this paper we obtain some results of harmonic analysis on quantum complex hyperbolic spaces. We introduce a quantum analog for the Laplace-Beltrami operator and its radial part. The latter appear to be second order $q$-difference operator, whose eigenfunctions are related to the Al-Salam-Chihara polynomials. We prove a Plancherel type theorem for it.
\end{abstract}

Key words: quantum groups, harmonic analysis on quantum symmetric spaces; $q$-difference operators; Al-Salam-Chihara polynomials; Plancherel formula

2010 Mathematics Subject Classification: 17B37; 20G42; 81R50; 33D45; 42C10

\section{Introduction}

Consider the group $S U_{n, m}$ and its homogeneous space $\mathcal{H}_{n, m}=S U_{n, m} / S\left(U_{n, m-1} \times U_{1}\right)$. The latter is called a complex hyperbolic space. The Faraut paper [5] on such pseudo-Hermitian symmetric spaces has a great impact into the theory of semisimple symmetric spaces of rank 1. Also there are numerous papers of Molchanov, van Dijk and others (see [11, 12, 21] and references therein) on representation theory related to these symmetric spaces and harmonic analysis on them. In particular, there is the celebrated Penrose transform which enables to relate classical bounded symmetric domains and complex hyperbolic spaces.

In this paper we develop harmonic analysis on quantum complex hyperbolic spaces. Recall that the related polynomial algebras were introduced in the early paper by Faddeev, Reshetikhin, and Takhtadjan [14]. Unfortunately, there was no further inquiry.

Nearly 10 years ago L. Vaksman and his team started the theory of quantum bounded symmetric domains. Their approach enables to formulate and solve various problems on noncommutative complex and harmonic analysis in these domains, geometric realizations of representations of quantum groups [19]. Their paper [17] establishes the missed link between quantum bounded symmetric domains and introduces this project.

The initial notions of function theory on quantum complex hyperbolic spaces $\mathcal{H}_{n, m}$ were introduced in [3]. Namely, a quantum analog $\mathcal{D}\left(\mathcal{H}_{n, m}\right)_{q, \mathfrak{k}}$ for the algebra of $U \mathfrak{k}=U \mathfrak{s}\left(\mathfrak{g l}_{\mathfrak{n}} \times \mathfrak{g l} \mathfrak{l}_{\mathfrak{m}}\right)$ finite smooth functions on $\mathcal{H}_{n, m}$ with compact support and an $U_{q} \mathfrak{s u}_{n, m}$-invariant integral $\int d \nu_{q}$ on it were constructed. We recall basic notations from the quantum group theory and [3] in Sections 2, 3, 4. Section 5 is devoted to the subalgebra of $U_{q} \mathfrak{k}$-invariant finite functions.

In Sections 6 and 7 we introduce a quantum analog $\square$ for the Laplace-Beltrami operator on $\mathcal{H}_{n, m}$. Also we consider its radial part $\square^{(0)}$, i.e., its restriction to the space $L^{2}\left(d \nu_{q}^{(0)}\right)$, where $\int \cdot d \nu_{q}^{(0)}$ is the restriction of $\int d \nu_{q}$ to the space of $U_{q} \mathfrak{k}$-invariant elements of $\mathcal{D}\left(\mathcal{H}_{n, m}\right)_{q, \mathfrak{k}}$. The

${ }^{\star}$ This paper is a contribution to the Special Issue "Relationship of Orthogonal Polynomials and Special Functions with Quantum Groups and Integrable Systems". The full collection is available at http://www.emis.de/journals/SIGMA/OPSF.html 
latter operator naturally appears to be $q$-difference operator and is related to a three-diagonal Jacobi matrix. Section 8 is devoted to generalized eigenfunctions of $\square^{(0)}$ and lead us towards an expected yet remarkable appearance of Al-Salam-Chihara polynomials. A spectral theorem for $\square^{(0)}$ in Section 9 is obtained as a corollary to well known results on these polynomials (see [9]).

\section{Preliminaries on quantum group theory}

Everywhere in the sequel we suppose $q \in(0,1)$. All algebras are associative and unital.

The Hopf algebra $U_{q} \mathfrak{s l}_{N}$ is given by its generators $K_{i}, K_{i}^{-1}, E_{i}, F_{i}, i=1,2, \ldots, N-1$, and the relations:

$$
\begin{aligned}
& K_{i} K_{j}=K_{j} K_{i}, \quad K_{i} K_{i}^{-1}=K_{i}^{-1} K_{i}=1, \\
& K_{i} E_{i}=q^{2} E_{i} K_{i}, \quad K_{i} F_{i}=q^{-2} F_{i} K_{i}, \\
& K_{i} E_{j}=q^{-1} E_{j} K_{i}, \quad K_{i} F_{j}=q F_{j} K_{i}, \quad|i-j|=1, \\
& E_{i} F_{j}-F_{j} E_{i}=\delta_{i j} \frac{K_{i}-K_{i}^{-1}}{q-q^{-1}}, \\
& E_{i}^{2} E_{j}-\left(q+q^{-1}\right) E_{i} E_{j} E_{i}+E_{j} E_{i}^{2}=0, \quad|i-j|=1, \\
& F_{i}^{2} F_{j}-\left(q+q^{-1}\right) F_{i} F_{j} F_{i}+F_{j} F_{i}^{2}=0, \quad|i-j|=1, \\
& {\left[E_{i}, E_{j}\right]=\left[F_{i}, F_{j}\right]=0, \quad|i-j| \neq 1 .}
\end{aligned}
$$

The comultiplication $\Delta$, the antipode $S$, and the counit $\varepsilon$ are defined on the generators by

$$
\begin{aligned}
& \Delta\left(E_{i}\right)=E_{i} \otimes 1+K_{i} \otimes E_{i}, \quad \Delta\left(F_{i}\right)=F_{i} \otimes K_{i}^{-1}+1 \otimes F_{i}, \quad \Delta\left(K_{i}\right)=K_{i} \otimes K_{i}, \\
& S\left(E_{i}\right)=-K_{i}^{-1} E_{i}, \quad S\left(F_{i}\right)=-F_{i} K_{i}, \quad S\left(K_{i}\right)=K_{i}^{-1}, \\
& \varepsilon\left(E_{i}\right)=\varepsilon\left(F_{i}\right)=0, \quad \varepsilon\left(K_{i}\right)=1,
\end{aligned}
$$

see [7, Chapter 4].

We need also the Hopf algebra $\mathbb{C}\left[S L_{N}\right]_{q}$ of matrix elements of finite dimensional weight $U_{q} \mathfrak{s l}_{N}$-modules. Recall that $\mathbb{C}\left[S L_{N}\right]_{q}$ can be defined by the generators $t_{i j}, i, j=1, \ldots, N$ (the matrix elements of the vector representation in a weight basis) and the relations

$$
\begin{array}{rlrl}
t_{i j^{\prime}} t_{i j^{\prime \prime}} & =q t_{i j^{\prime \prime}} t_{i j^{\prime}}, & & j^{\prime}<j^{\prime \prime}, \\
t_{i^{\prime} j} t_{i^{\prime \prime} j} & =q t_{i^{\prime \prime} j} t_{i^{\prime} j}, & & i^{\prime}<i^{\prime \prime}, \\
t_{i j} t_{i^{\prime} j^{\prime}}=t_{i^{\prime} j^{\prime}} t_{i j}, & & i<i^{\prime} \& j>j^{\prime}, \\
t_{i j} t_{i^{\prime} j^{\prime}}=t_{i^{\prime} j^{\prime}} t_{i j}+\left(q-q^{-1}\right) t_{i j^{\prime}} t_{i^{\prime} j}, & & i<i^{\prime} \& j<j^{\prime},
\end{array}
$$

together with one more relation

$$
\operatorname{det}_{q} \mathbf{t}=1
$$

where $\operatorname{det}_{q} \mathbf{t}$ is a $q$-determinant of the matrix $\mathbf{t}=\left(t_{i j}\right)_{i, j=1, \ldots, N}$ :

$$
\operatorname{det}_{q} \mathbf{t}=\sum_{s \in S_{N}}(-q)^{l(s)} t_{1 s(1)} t_{2 s(2)} \cdots t_{N s(N)},
$$

with $l(s)=\operatorname{card}\{(i, j) \mid i<j \& s(i)>s(j)\}$. The algebra $\mathbb{C}\left[S L_{N}\right]_{q}$ is endowed with the standard structure of $U_{q}^{\mathrm{op}} \mathfrak{s l}_{N} \otimes U_{q} \mathfrak{s l}_{N}$-module algebra (here 'op' reflects the fact that we should change the multiplication by the opposite one). 
Let also $U_{q} \mathfrak{s u}_{n, m}, m+n=N$, denotes the Hopf $*$-algebra $\left(U_{q} \mathfrak{s l}_{N}, *\right)$ given by

$$
\left(K_{j}^{ \pm 1}\right)^{*}=K_{j}^{ \pm 1}, \quad E_{j}^{*}=\left\{\begin{array}{ll}
K_{j} F_{j}, & j \neq n, \\
-K_{j} F_{j}, & j=n,
\end{array} \quad F_{j}^{*}= \begin{cases}E_{j} K_{j}^{-1}, & j \neq n, \\
-E_{j} K_{j}^{-1}, & j=n,\end{cases}\right.
$$

with $j=1, \ldots, N-1[14,15]$.

Recall the notion of an algebra of 'regular functions on the quantum principal homogeneous space' $X$ constructed in [15]. $\operatorname{Put} \operatorname{Pol}(\tilde{X})_{q} \stackrel{\text { def }}{=}\left(\mathbb{C}\left[S L_{N}\right]_{q}, *\right)$, where the involution $*$ is defined by

$$
t_{i j}^{*}=\operatorname{sign}[(i-m-1 / 2)(n-j+1 / 2)](-q)^{j-i} \operatorname{det}_{q} T_{i j} .
$$

Here $\operatorname{det}_{q}$ is the quantum determinant [4], and the matrix $T_{i j}$ is derived from the matrix $\mathbf{t}=\left(t_{k l}\right)$ by discarding its $i$ 's row and $j$ 's column. In [15] it is proved that $\operatorname{Pol}(\widetilde{X})_{q}$ is a $U_{q} \mathfrak{s u} n_{n, m}$-module *-algebra.

\section{$3 \quad$ A *-algebra $\operatorname{Pol}\left(\mathcal{H}_{n, m}\right)_{q}$}

Let $m, n \in \mathbb{N}, m \geq 2$, and $N \stackrel{\text { def }}{=} n+m$. Recall that the classical complex hyperbolic space $\mathcal{H}_{n, m}$ can be obtained by projectivization of the domain

$$
\widehat{\mathcal{H}}_{n, m}=\left\{\left.\left(t_{1}, \ldots, t_{N}\right) \in \mathbb{C}^{N}\left|-\sum_{j=1}^{n}\right| t_{j}\right|^{2}+\sum_{j=n+1}^{N}\left|t_{j}\right|^{2}>0\right\} .
$$

Now we pass from the classical case $q=1$ to the quantum case $0<q<1$. Let us consider the well known [14] $q$-analog of the polynomial algebras. Let $\operatorname{Pol}\left(\widehat{\mathcal{H}}_{n, m}\right)_{q}$ be the unital $*$-algebra with the generators $t_{1}, t_{2}, \ldots, t_{N}$ and the commutation relations as follows:

$$
\begin{aligned}
& t_{i} t_{j}=q t_{j} t_{i}, \quad i<j, \\
& t_{i} t_{j}^{*}=q t_{j}^{*} t_{i}, \quad i \neq j, \\
& t_{i} t_{i}^{*}=t_{i}^{*} t_{i}+\left(q^{-2}-1\right) \sum_{k=i+1}^{N} t_{k} t_{k}^{*}, \quad i>n, \\
& t_{i} t_{i}^{*}=t_{i}^{*} t_{i}+\left(q^{-2}-1\right) \sum_{k=i+1}^{n} t_{k} t_{k}^{*}-\left(q^{-2}-1\right) \sum_{k=n+1}^{N} t_{k} t_{k}^{*}, \quad i \leq n .
\end{aligned}
$$

Obviously,

$$
c=-\sum_{j=1}^{n} t_{j} t_{j}^{*}+\sum_{j=n+1}^{N} t_{j} t_{j}^{*}
$$

is central in $\operatorname{Pol}\left(\widehat{\mathcal{H}}_{n, m}\right)_{q}$. Moreover, $c$ is not a zero divisor in $\operatorname{Pol}\left(\widehat{\mathcal{H}}_{n, m}\right)_{q}$. This allows one to embed the $*$-algebra $\operatorname{Pol}\left(\widehat{\mathcal{H}}_{n, m}\right)_{q}$ into its localization $\operatorname{Pol}\left(\widehat{\mathcal{H}}_{n, m}\right)_{q, c}$ with respect to the multiplicative system $c^{\mathbb{N}}$.

The *-algebra $\operatorname{Pol}\left(\widehat{\mathcal{H}}_{n, m}\right)_{q, c}$ admits the following bigrading:

$$
\operatorname{deg} t_{j}=(1,0), \quad \operatorname{deg} t_{j}^{*}=(0,1), \quad j=1,2, \ldots, N .
$$

Introduce the notation

$$
\operatorname{Pol}\left(\mathcal{H}_{n, m}\right)_{q}=\left\{f \in \operatorname{Pol}\left(\widehat{\mathcal{H}}_{n, m}\right)_{q, c} \mid \operatorname{deg} f=(0,0)\right\} .
$$


This *-algebra $\operatorname{Pol}\left(\mathcal{H}_{n, m}\right)_{q}$ will be called the algebra of regular functions on the quantum hyperbolic space.

We are going to endow the $*$-algebra $\operatorname{Pol}\left(\mathcal{H}_{n, m}\right)_{q}$ with a structure of $U_{q} \mathfrak{s u}$, bra [4]. For this purpose, we embed it into the $U_{q} \mathfrak{s u}_{n, m}$-module $*$-algebra $\operatorname{Pol}(\widetilde{X})_{q}$.

By a $q$-analog of the Laplace expansion of $\operatorname{det}_{q} \mathbf{t}$ along the first row [8, Section 9.2] and (1), one can obtain from $\operatorname{det}_{q} \mathbf{t}=1$ that

$$
-\sum_{j=1}^{n} t_{1 j} t_{1 j}^{*}+\sum_{j=n+1}^{N} t_{1 j} t_{1 j}^{*}=1 .
$$

Thus the map $J: t_{j} \mapsto t_{1 j}, j=1,2, \ldots, N$, admits a unique extension to a homomorphism of *-algebras $J: \operatorname{Pol}\left(\widehat{\mathcal{H}}_{n, m}\right)_{q, c} \rightarrow \operatorname{Pol}(\widetilde{X})_{q}$. Its image will be denoted by $\operatorname{Pol}\left(\widetilde{\mathcal{H}}_{n, m}\right)_{q}$. It is easy to verify that the $*$-algebra $\operatorname{Pol}\left(\mathcal{H}_{n, m}\right)_{q}$ is embedded this way into $\operatorname{Pol}\left(\widetilde{\mathcal{H}}_{n, m}\right)_{q}$ and its image is just the subalgebra in $\operatorname{Pol}\left(\widetilde{\mathcal{H}}_{n, m}\right)_{q}$ generated by $t_{1 j} t_{1 k}^{*}, j, k=1,2, \ldots, N$ (recall that $c$ goes to $\operatorname{det}_{q} \mathbf{t}=1$ ). In what follows we will identify $\operatorname{Pol}\left(\mathcal{H}_{n, m}\right)_{q}$ with its image under the map $J$.

Consider the subalgebra $U_{q} \mathfrak{s}\left(\mathfrak{g l}_{1} \times \mathfrak{g l}_{N-1}\right)$ generated by $K_{i}^{ \pm 1}, i=1, \ldots, N-1, E_{j}, F_{j}$, $j=2, \ldots, N-1$. By obvious reasons,

$$
\operatorname{Pol}\left(\mathcal{H}_{n, m}\right)_{q}=\left\{f \in \operatorname{Pol}(\tilde{X})_{q} \mid L(\xi) f=\varepsilon(\xi) f, \xi \in U_{q} \mathfrak{s}\left(\mathfrak{g l}_{1} \times \mathfrak{g l}_{N-1}\right)\right\}
$$

where $L$ is the left action of $U_{q}^{\mathrm{op}} \mathfrak{s l}_{N}$ in $\operatorname{Pol}(\widetilde{X})_{q}$.

Let $I_{\varphi}, \varphi \in \mathbb{R} / 2 \pi \mathbb{Z}$, be the $*$-automorphism of the $*$-algebra $\operatorname{Pol}\left(\widetilde{\mathcal{H}}_{n, m}\right)_{q}$ defined on the generators $\left\{t_{j}\right\}_{j=1, \ldots, N}$ by

$$
I_{\varphi}: t_{j} \mapsto e^{i \varphi} t_{j}
$$

We use the notation $t_{j}$ instead of $t_{1 j}$ for the generators of $\operatorname{Pol}\left(\widetilde{\mathcal{H}}_{n, m}\right)_{q}$.

Then one more description of $\operatorname{Pol}\left(\mathcal{H}_{n, m}\right)_{q}$ is as follows:

$$
\operatorname{Pol}\left(\widetilde{\mathcal{H}}_{n, m}\right)_{q}=\left\{f \in \operatorname{Pol}\left(\widetilde{\mathcal{H}}_{n, m}\right)_{q} \mid I_{\varphi}(f)=f \text { for all } \varphi\right\}
$$

At the end of this section we list explicit formulas for the action of $U_{q} \mathfrak{s u}_{n, m}$ on $\operatorname{Pol}\left(\widetilde{\mathcal{H}}_{n, m}\right)$ :

$$
\begin{aligned}
& E_{j} t_{i}=\left\{\begin{array}{ll}
q^{-1 / 2} t_{i-1}, & j+1=i, \\
0, & \text { otherwise },
\end{array} \quad F_{j} t_{i}= \begin{cases}q^{1 / 2} t_{i+1}, & j=i, \\
0, & \text { otherwise },\end{cases} \right. \\
& K_{j}^{ \pm 1} t_{i}= \begin{cases}q^{ \pm 1} t_{i}, & j=i, \\
q^{\mp 1} t_{i}, & j+1=i, \\
t_{i}, & \text { otherwise. }\end{cases}
\end{aligned}
$$

\section{Algebras of generalized and finite functions on the quantum $\mathcal{H}_{n, m}$}

Let us construct a faithful *-representation $T$ of $\operatorname{Pol}\left(\mathcal{H}_{n, m}\right)_{q}$ in a pre-Hilbert space $\mathcal{H}$ (our method is well known; see, for example, [15]).

The space $\mathcal{H}$ is a linear span of its orthonormal basis $\left\{e\left(i_{1}, i_{2}, \ldots, i_{N-1}\right) \mid i_{1}, \ldots, i_{n} \in-\mathbb{Z}_{+}\right.$; $\left.i_{n+1}, \ldots, i_{N-1} \in \mathbb{N}\right\}$. 
The *-representation $T$ is a restriction to $\operatorname{Pol}\left(\mathcal{H}_{n, m}\right)_{q}$ of the $*$-representation of $\operatorname{Pol}\left(\widetilde{\mathcal{H}}_{n, m}\right)_{q}$ defined by

$$
\begin{aligned}
& T\left(t_{j}\right) e\left(i_{1}, \ldots, i_{N-1}\right)=q^{\sum_{k=1}^{j-1} i_{k}}\left(q^{2\left(i_{j}-1\right)}-1\right)^{1 / 2} e\left(i_{1}, \ldots, i_{j}-1, \ldots, i_{N-1}\right), \\
& T\left(t_{j}^{*}\right) e\left(i_{1}, \ldots, i_{N-1}\right)=q^{\sum_{k=1}^{j-1} i_{k}}\left(q^{2 i_{j}}-1\right)^{1 / 2} e\left(i_{1}, \ldots, i_{j}+1, \ldots, i_{N-1}\right),
\end{aligned}
$$

for $j \leq n$,

$$
\begin{aligned}
& T\left(t_{j}\right) e\left(i_{1}, \ldots, i_{N-1}\right)=q^{\sum_{k=1}^{j-1} i_{k}}\left(1-q^{2\left(i_{j}-1\right)}\right)^{1 / 2} e\left(i_{1}, \ldots, i_{j}-1, \ldots, i_{N-1}\right), \\
& T\left(t_{j}^{*}\right) e\left(i_{1}, \ldots, i_{N-1}\right)=q^{\sum_{k=1}^{j-1} i_{k}}\left(1-q^{2 i_{j}}\right)^{1 / 2} e\left(i_{1}, \ldots, i_{j}+1, \ldots, i_{N-1}\right),
\end{aligned}
$$

for $n<j<N$, and, finally,

$$
\begin{aligned}
& T\left(t_{N}\right) e\left(i_{1}, \ldots, i_{N-1}\right)=q^{\sum_{k=1}^{N-1} i_{k}} e\left(i_{1}, \ldots, i_{N-1}\right), \\
& T\left(t_{N}^{*}\right) e\left(i_{1}, \ldots, i_{N-1}\right)=q^{\sum_{k=1}^{N-1} i_{k}} e\left(i_{1}, \ldots, i_{N-1}\right) .
\end{aligned}
$$

Define the elements $\left\{x_{j}\right\}_{j=1, \ldots, N}$ as follows:

$$
x_{j} \stackrel{\text { def }}{=} \begin{cases}\sum_{k=j}^{N} t_{k} t_{k}^{*}, & j>n, \\ -\sum_{k=j}^{n} t_{k} t_{k}^{*}+\sum_{k=n+1}^{N} t_{k} t_{k}^{*}, & j \leq n .\end{cases}
$$

Obviously, $x_{1}=1, x_{i} x_{j}=x_{j} x_{i}$,

$$
t_{j} x_{k}= \begin{cases}q^{2} x_{k} t_{j}, & j<k \\ x_{k} t_{j}, & j \geq k\end{cases}
$$

The vectors $e\left(i_{1}, \ldots, i_{N-1}\right)$ are joint eigenvectors of the operators $T\left(x_{j}\right), j=1,2, \ldots, N$ :

$$
T\left(x_{j}\right) e\left(i_{1}, \ldots, i_{N-1}\right)=q^{2 \sum_{k=1}^{j-1} i_{k}} e\left(i_{1}, \ldots, i_{N-1}\right) .
$$

The joint spectrum of the pairwise commuting operators $T\left(x_{j}\right), j=1,2, \ldots, N$, is

$$
\begin{aligned}
\mathfrak{M}=\{ & \left(x_{1}, \ldots, x_{N}\right) \in \mathbb{R}^{N} \mid x_{i} / x_{j} \in q^{2 \mathbb{Z}} \& 1=x_{1} \leq x_{2} \leq \cdots \leq x_{n+1}, \\
& \left.\& x_{n+1}>x_{n+2}>\cdots>x_{N}>0\right\} .
\end{aligned}
$$

The next proposition was proved in [3].

Proposition 1. $T$ is a faithful representation of $\operatorname{Pol}\left(\mathcal{H}_{n, m}\right)_{q}$.

Let us now introduce the notion of generalized functions on the quantum complex hyperbolic space $\mathcal{H}_{n, m}$. Evidently, using the commutation relations, one can decompose every polynomial $f \in \operatorname{Pol}\left(\widetilde{\mathcal{H}}_{n, m}\right)_{q}$ as follows:

$$
f=\sum_{I=\left(i_{1}, \ldots, i_{N}\right), J=\left(j_{1}, \ldots, j_{N}\right) \in \mathbb{Z}_{+}^{N}} c_{I J} t_{1}^{i_{1}} \cdots t_{n}^{i_{n}} t_{n+1}^{* i_{n+1}} \cdots t_{N}^{* i_{N}} t_{N}^{j_{N}} \cdots t_{n+1}^{j_{n+1}} t_{n}^{* j_{n}} \cdots t_{1}^{* j_{1}}, \quad c_{I J} \in \mathbb{C} .
$$


Due to (3), the latter can be reduced to the decomposition

$$
f=\sum_{\left(i_{1}, \ldots, i_{N}, j_{1}, \ldots, j_{N}\right): i_{k} j_{k}=0} t_{1}^{i_{1}} \cdots t_{n}^{i_{n}} t_{n+1}^{* i_{n+1}} \cdots t_{N}^{* i_{N}} f_{I J}\left(x_{2}, \ldots, x_{N}\right) t_{N}^{j_{N}} \cdots t_{n+1}^{j_{n+1}} t_{n}^{* j_{n}} \cdots t_{1}^{* j_{1}}
$$

where $f_{I J}\left(x_{2}, \ldots, x_{N}\right)$ are polynomials.

One can equip $\operatorname{Pol}\left(\widetilde{\mathcal{H}}_{n, m}\right)_{q}$ with the weakest topology such that the functionals

$$
l_{\left(i_{1}, \ldots, i_{N} ; j_{1}, \ldots, j_{N}\right)}(f)=\left(T(f) e\left(i_{1}, \ldots, i_{N}\right), e\left(j_{1}, \ldots, j_{N}\right)\right)
$$

are continuous. The completion of $\operatorname{Pol}\left(\widetilde{\mathcal{H}}_{n, m}\right)_{q}$ w.r.t. this topology will be considered as the space of generalized functions on the quantum $\widehat{\mathcal{H}}_{n, m}$ and denoted by $\mathcal{D}\left(\widetilde{\mathcal{H}}_{n, m}\right)_{q}^{\prime}$. Naturally, one can extend $T$ to a representation of $\mathcal{D}\left(\widetilde{\mathcal{H}}_{n, m}\right)_{q}^{\prime}$ by continuity. Now (6) allows one to identify $\mathcal{D}\left(\widetilde{\mathcal{H}}_{n, m}\right)_{q}^{\prime}$ with the space of formal series

$$
f=\sum_{\left(i_{1}, \ldots, i_{N}, j_{1}, \ldots, j_{N}\right): i_{k} j_{k}=0} t_{1}^{i_{1}} \cdots t_{n}^{i_{n}} t_{n+1}^{* i_{n+1}} \cdots t_{N}^{* i_{N}} f_{I J}\left(x_{2}, \ldots, x_{N}\right) t_{N}^{j_{N}} \cdots t_{n+1}^{j_{n+1}} t_{n}^{* j_{n}} \cdots t_{1}^{* j_{1}}
$$

where $f_{I J}\left(x_{2}, \ldots, x_{N}\right)$ are functions on $\mathfrak{M}$. The topology on this space of formal series is the topology of pointwise convergence of the functions $f_{I J}$.

Denote by $f_{0}$ the following function

$$
f_{0}=f_{0}\left(x_{n+1}\right)= \begin{cases}1, & x_{n+1}=1 \\ 0, & x_{n+1} \in q^{-2 \mathbb{N}}\end{cases}
$$

(Recall that $\operatorname{spec} x_{n+1}=q^{-2 \mathbb{Z}_{+}}$.) Thus $f_{0}$ is a $q$-analog of the characteristic function of the submanifold

$$
\left\{\left(t_{1}, \ldots, t_{N}\right) \in \mathbb{C}^{N} \mid t_{1}=t_{2}=\cdots=t_{n}=0\right\} \cap \mathcal{H}_{n, m} .
$$

Introduce now a $*$-algebra $\operatorname{Fun}\left(\widetilde{\mathcal{H}}_{n, m}\right)_{q} \subset \mathcal{D}\left(\widetilde{\mathcal{H}}_{n, m}\right)_{q}^{\prime}$ generated by $\operatorname{Pol}\left(\widetilde{\mathcal{H}}_{n, m}\right)_{q}$ and $f_{0}$. Easy computations from $(4)$ show that $f_{0}$ satisfies the following relations:

$$
\begin{aligned}
& t_{j}^{*} f_{0}=f_{0} t_{j}=0, \quad j \leq n, \\
& x_{n+1} f_{0}=f_{0} x_{n+1}=f_{0}, \\
& f_{0}^{2}=f_{0}^{*}=f_{0}, \\
& t_{j} f_{0}=f_{0} t_{j}, \quad t_{j}^{*} f_{0}=f_{0} t_{j}^{*}, \quad j \geq n+1 .
\end{aligned}
$$

The relation $I_{\varphi} f_{0}=f_{0}$ allows one to extend the $*$-automorphism $I_{\varphi}(2)$ of the algebra $\operatorname{Pol}\left(\widetilde{\mathcal{H}}_{n, m}\right)_{q}$ to the $*$-automorphism of $\operatorname{Fun}\left(\widetilde{\mathcal{H}}_{n, m}\right)_{q}$. Let

$$
\operatorname{Fun}\left(\mathcal{H}_{n, m}\right)_{q} \stackrel{\text { def }}{=}\left\{f \in \operatorname{Fun}\left(\widetilde{\mathcal{H}}_{n, m}\right)_{q} \mid I_{\varphi} f=f\right\} .
$$

Obviously, there exists a unique extension of the $*$-representation $T$ to a $*$-representation of the *-algebra $\operatorname{Fun}\left(\mathcal{H}_{n, m}\right)_{q}$ such that $T\left(f_{0}\right)$ is the orthogonal projection of $\mathcal{H}$ onto the linear span of vectors $\left\{e(\underbrace{0, \ldots, 0}_{n}, i_{n+1}, \ldots, i_{N-1}) \mid i_{n+1}, \ldots, i_{N-1} \in \mathbb{N}\right\}$.

Let $\mathcal{D}\left(\mathcal{H}_{n, m}\right)_{q, \mathfrak{k}}$ be the two-sided ideal of $\operatorname{Fun}\left(\mathcal{H}_{n, m}\right)_{q}$ generated by $f_{0}$. We call this ideal the algebra of finite functions on the quantum hyperbolic space. It is a quantum analog for the algebra of $U \mathfrak{k}=U \mathfrak{s}\left(\mathfrak{g l}_{n} \times \mathfrak{g l}_{m}\right)$-finite smooth functions on $\mathcal{H}_{n, m}$ with compact support. 
Remark 1. Let us explain the adjective 'finite'. If $f$ is a finite function, $T(f)$ is an operator with only a finite number of nonzero entries. However, we do not consider all possible finite functions (and, therefore, all operators with finite number of nonzero entries) but only $U_{q} \mathfrak{k}$-finite ones, cf. [5].

It was proved in [3] that

Theorem 1. The representation $T$ of $\mathcal{D}\left(\mathcal{H}_{n, m}\right)_{q, \mathfrak{k}}$ is faithful.

Remark 2. Let $f\left(x_{n+1}\right)$ be a polynomial. Then it follows from (3), (4) that

$$
\sum_{i=1}^{n} t_{i} f\left(x_{n+1}\right) t_{i}^{*}=f\left(q^{2} x_{n+1}\right) \sum_{i=1}^{n} t_{i} t_{i}^{*}=f\left(q^{2} x_{n+1}\right)\left(x_{n+1}-1\right) .
$$

This computation, together with (7), allows one to consider the element $f_{1}=\sum_{i=1}^{n} t_{i} f_{0} t_{i}^{*}$ as a function of $x_{n+1}$ such that

$$
f_{1}\left(x_{n+1}\right)= \begin{cases}q^{-2}-1, & x_{n+1}=q^{-2} \\ 0, & x_{n+1}=1 \text { or } x_{n+1} \in q^{-2 \mathbb{N}-2} .\end{cases}
$$

Thus a multiple application of (8) leads to the following claim: $\mathcal{D}\left(\mathcal{H}_{n, m}\right)_{q, \mathfrak{k}}$ contains all finite functions of $x_{n+1}$ (i.e., such functions $f$ that $f\left(q^{-n}\right)=0$ for all but finitely many $n \in \mathbb{N}$ ).

The $U_{q} \mathfrak{s u} u_{n, m}$-module algebra structure is established on $\mathcal{D}\left(\mathcal{H}_{n, m}\right)_{q, \mathfrak{k}}$ by the following:

$$
\begin{aligned}
& E_{n} f_{0}=-\frac{q^{-1 / 2}}{q^{-2}-1} t_{n} f_{0} t_{n+1}^{*}, \quad F_{n} f_{0}=-\frac{q^{3 / 2}}{q^{-2}-1} t_{n+1} f_{0} t_{n}^{*}, \quad K_{n} f_{0}=f_{0}, \\
& E_{j} f_{0}=F_{j} f_{0}=\left(K_{j}-1\right) f_{0}=0, \quad j \neq n .
\end{aligned}
$$

Now we present an explicit formula for a positive invariant integral on the space of finite functions $\mathcal{D}\left(\mathcal{H}_{n, m}\right)_{q, \mathfrak{k}}$ and thereby establish its existence.

Let $\nu_{q}: \mathcal{D}\left(\mathcal{H}_{n, m}\right)_{q, \mathfrak{k}} \rightarrow \mathbb{C}$ be a linear functional defined by

$$
\nu_{q}(f)=\operatorname{Tr}(T(f) \cdot Q)=\int_{\mathcal{H}_{n, m}} f d \nu_{q}
$$

where $Q: \mathcal{H} \rightarrow \mathcal{H}$ is the linear operator given on the basis elements $e\left(i_{1}, \ldots, i_{N-1}\right)$ by

$$
Q e\left(i_{1}, \ldots, i_{N-1}\right)=\text { const }_{1} q^{2 \sum_{j=1}^{N-1}(N-j) i_{j}} e\left(i_{1}, \ldots, i_{N-1}\right), \quad \text { const }_{1}>0 .
$$

Theorem 2 ([3]). The functional $\nu_{q}$ is well defined, positive, and $U_{q} \mathfrak{s u}_{n, m}$-invariant.

One has to normalize this integral in a some way. In [3] we put $\int_{\mathcal{H}_{n, m}} f_{0} d \nu_{q}=1$, so the constant in the previous theorem equals

$$
\text { const }_{1}=\prod_{j=1}^{m-1}\left(q^{-2 j}-1\right)
$$




\section{The subalgebra $\mathcal{D}\left(\mathcal{H}_{n, m}\right)_{q, \mathfrak{k}}^{U_{q} \mathfrak{k}}$}

In this section we restrict ourselves to subalgebras of $U_{q} \mathfrak{k}$-invariant elements. It is well known that $\mathcal{H}_{n, m}$ is a pseudo-Hermitian symmetric space of rank 1 [12]. The following proposition is a natural quantum analog for this fact.

Proposition 2. $\mathcal{D}\left(\mathcal{H}_{n, m}\right)_{q, \mathfrak{k}}^{U_{q} \mathfrak{k}}=\left\{f\left(x_{n+1}\right) \in \mathcal{D}\left(\mathcal{H}_{n, m}\right)_{q, \mathfrak{k}}\right\}$.

Since $U_{q} \mathfrak{k}=\mathbb{C}\left[K_{n}, K_{n}^{-1}\right] \otimes U_{q} \mathfrak{s l}_{n} \otimes U_{q} \mathfrak{s l}_{m}$, the proof of this proposition follows from the next statement on $U_{q} \mathfrak{s l}_{n} \times U_{q} \mathfrak{s l}_{m}$-isotypic components in $\mathcal{D}\left(\mathcal{H}_{n, m}\right)_{q, \mathfrak{k}}$.

Proposition 3. $U_{q} \mathfrak{s l}_{n} \times U_{q} \mathfrak{s l}_{m}$-isotypic components of $\mathcal{D}\left(\mathcal{H}_{n, m}\right)_{q, \mathfrak{k}}$ correspond to the modules $L^{(n)}\left(a \varpi_{1}+d \varpi_{n-1}\right) \otimes L^{(m)}\left(c \varpi_{1}+b \varpi_{m-1}\right)$ with $a, b, c, d \in \mathbb{Z}_{+}, a+c=b+d$ (with infinite multiplicity). The highest weight subspace is spanned by the vectors $t_{1}^{a} t_{N}^{* b} \varphi\left(x_{n+1}\right) t_{n+1}^{c} t_{n}^{* d}$, where $\varphi\left(x_{n+1}\right)$ is a finite function.

Remark 3. The sign $\otimes$ reflects the fact that the multipliers are modules of different algebras $U_{q} \mathfrak{s l}_{n}$ and $U_{q} \mathfrak{s l}_{m}$.

Proof. Let us describe $U_{q} \mathfrak{s l}_{n} \times U_{q} \mathfrak{s l}_{m}$-highest weight vectors in $\mathcal{D}\left(\mathcal{H}_{n, m}\right)_{q, \mathfrak{k}}$. One can decompose every finite function $f \in \mathcal{D}\left(\mathcal{H}_{n, m}\right)_{q, \mathfrak{k}}$ in the following way

$$
f=\sum_{\substack{I=\left(i_{1}, \ldots, i_{N}\right), J=\left(j_{1}, \ldots, j_{N}\right) \in \mathbb{Z}_{+}^{N} \\ i_{1}+\cdots+i_{n}+j_{n+1}+\cdots+j_{N} \\=i_{n+1}+\cdots+i_{N}+j_{1}+\cdots+j_{n}}} c_{I J} t_{1}^{i_{1}} \cdots t_{n}^{i_{n}} t_{n+1}^{* i_{n+1}} \cdots t_{N}^{* i_{N}} f_{0} t_{N}^{j_{N}} \cdots t_{n+1}^{j_{n+1}} t_{n}^{* j_{n}} \cdots t_{1}^{* j_{1}}, \quad c_{I J} \in \mathbb{C} .
$$

(just by applying the commutation relations, cf. (5)). Let $L^{(n)}(\lambda)$ be the finite dimensional $U_{q} \mathfrak{s l}_{n^{-}}$ module with highest weight $\lambda$ and $\varpi_{j}$ are fundamental weights of $\mathfrak{s l}_{n}$. By standard arguments,

$$
\begin{aligned}
& \text { l.s. }\left\{t_{1}^{l_{1}} t_{2}^{l_{2}} \cdots t_{n}^{l_{n}} \mid l_{1}+\cdots+l_{n}=a\right\}=L^{(n)}\left(a \varpi_{1}\right), \\
& \text { l.s. }\left\{t_{1}^{* l_{1}} t_{2}^{* l_{2}} \cdots t_{n}^{* l_{n}} \mid l_{1}+\cdots+l_{n}=a\right\}=L^{(n)}\left(a \varpi_{n-1}\right), \\
& \text { l.s. }\left\{t_{n+1}^{l_{n+1}} t_{n+2}^{l_{n+2}} \cdots t_{N}^{l_{N}} \mid l_{n+1}+\cdots+l_{N}=a\right\}=L^{(m)}\left(a \varpi_{1}\right), \\
& \text { l.s. }\left\{t_{n+1}^{* l_{n+1}} t_{n+2}^{* l_{n+2}} \cdots t_{N}^{* l_{N}} \mid l_{n+1}+\cdots+l_{N}=a\right\}=L^{(m)}\left(a \varpi_{m-1}\right) .
\end{aligned}
$$

Thus we have an epimorphism

$$
\begin{aligned}
& \bigoplus_{a+c=b+d} L^{(n)}\left(a \varpi_{1}\right) \otimes L^{(m)}\left(d \varpi_{m-1}\right) \otimes L^{(m)}\left(c \varpi_{1}\right) \otimes L^{(n)}\left(b \varpi_{n-1}\right) \rightarrow \mathcal{D}\left(\mathcal{H}_{n, m}\right)_{q, \mathfrak{k}}, \\
& t_{1}^{i_{1}} \cdots t_{n}^{i_{n}} \otimes t_{n+1}^{* i_{n+1}} \cdots t_{N}^{* i_{N}} \otimes t_{N}^{j_{N}} \cdots t_{n+1}^{j_{n+1}} \otimes t_{n}^{* j_{n}} \cdots t_{1}^{* j_{1}} \\
& \quad \mapsto t_{1}^{i_{1}} \cdots t_{n}^{i_{n}} t_{n+1}^{* i_{n+1}} \cdots t_{N}^{* i_{N}} f_{0} t_{N}^{j_{N}} \cdots t_{n+1}^{j_{n+1}} t_{n}^{* j_{n}} \cdots t_{1}^{* j_{1}} .
\end{aligned}
$$

Recall that, as in the classical case, see [13],

$$
L^{(n)}\left(a \varpi_{1}\right) \otimes L^{(n)}\left(b \varpi_{n-1}\right)=\oplus_{i=0}^{\max (a, b)} L^{(n)}\left((a-i) \varpi_{1}+(b-i) \varpi_{n-1}\right)
$$

in the category of $U_{q} \mathfrak{s l}_{n}$-modules. By explicit calculations one can show that the $U_{q} \mathfrak{s l}_{n}$-highest weight vector in $L^{(n)}\left((a-i) \varpi_{1}+(b-i) \varpi_{n-1}\right) \subset L^{(n)}\left(a \varpi_{1}\right) \otimes L^{(n)}\left(b \varpi_{n-1}\right)$ has the form

$$
t_{1}^{a-i}\left(\sum_{j=1}^{n} t_{j} \otimes t_{j}^{*}\right)^{i} t_{n}^{*(b-i)}
$$

Now a routine application of the commutation relations (similar to the ones in Remark 2) allows one to reduce every highest weight vector to a linear span of the vectors $t_{1}^{a} t_{N}^{* b} \varphi\left(x_{n+1}\right) t_{n+1}^{c} t_{n}^{* d}$, where $\varphi\left(x_{n+1}\right)$ is a finite function. 
Now let us obtain an explicit form of the restriction of $\nu_{q}$ to the space $\mathcal{D}\left(\mathcal{H}_{n, m}\right)_{q, \mathfrak{k}}^{U_{q} \mathfrak{k}}$ of $U_{q} \mathfrak{k}-$ invariant elements of $\mathcal{D}\left(\mathcal{H}_{n, m}\right)_{q, \mathfrak{k}}$.

Recall the standard notation $(a ; q)_{k}=(1-a) \cdots\left(1-a q^{k-1}\right),(a ; q)_{0}=1$,

$$
\int_{1}^{\infty} f(x) d_{q^{-2}} x=\left(q^{-2}-1\right) \sum_{k=0}^{\infty} f\left(q^{-2 k}\right) q^{-2 k} .
$$

Proposition 4. For any function $f\left(x_{n+1}\right) \in \mathcal{D}\left(\mathcal{H}_{n, m}\right)_{q, \mathfrak{k}}$ one has

$$
\int_{\mathcal{H}_{n, m}} f\left(x_{n+1}\right) d \nu_{q}=\int_{1}^{\infty} f(x) \rho(x) d_{q^{-2}} x,
$$

where

$$
\rho(x)=\text { const }_{2} x^{m-1}\left(q^{-2} x-1\right)\left(q^{-4} x-1\right) \cdots\left(q^{-2(n-1)} x-1\right),
$$

and const $_{2}=\frac{1}{q^{-2}-1} \prod_{j=1}^{n-1} \frac{1}{q^{-2 j}-1}$.

Proof. By explicit calculations,

$$
\begin{aligned}
& \int_{\mathcal{H}_{n, m}} f\left(x_{n+1}\right) d \nu_{q}=\text { const }_{1} \sum_{\substack{i_{1} \ldots, i_{n} \in-\mathbb{Z}_{+}, i_{n+1}, \ldots, i_{N-1} \in \mathbb{N}}} f\left(q^{2 i_{1}+\cdots+2 i_{n}}\right) q^{2(N-1) i_{1}+2(N-2) i_{2}+\cdots+2 i_{N-1}} \\
& =\text { const }_{1} \sum_{i_{n+1}, \ldots, i_{N-1}=1}^{\infty}\left(\sum_{\substack{i_{1}, \ldots, i_{n} \in-\mathbb{Z}_{+}, i_{1}+\cdots+i_{n}=i}} f\left(q^{2 i}\right) q^{2(N-1) i_{1}+\cdots+2 m i_{n}}\right) q^{2(m-1) i_{n+1}+\cdots+2 i_{N-1}} \\
& =\text { const }_{1} \sum_{i \in-\mathbb{Z}_{+}} \frac{q^{m(m-1)}}{\left(q^{2} ; q^{2}\right)_{m-1}}\left(\sum_{\substack{i_{1}, \ldots, i_{n-1} \in-\mathbb{Z}_{+}, i_{1}+\cdots+i_{n-1} \geq i}} q^{2(n-1) i_{1}+\cdots+2 i_{n-1}}\right) f\left(q^{2 i}\right) q^{2 m i} \\
& =\operatorname{const}_{1} \frac{q^{m(m-1)}}{\left(q^{2} ; q^{2}\right)_{m-1}} \sum_{i \in-\mathbb{Z}_{+}} f\left(q^{2 i}\right) q^{2 m i}\left(\sum_{\substack{i_{1}, \ldots, i_{n-1} \in \mathbb{Z}_{+}, i_{1}+\cdots+i_{n-1} \leq-i}} q^{-2(n-1) i_{1}-\cdots-2 i_{n-1}}\right) \text {. }
\end{aligned}
$$

Let us verify that for every $j, k \in \mathbb{Z}_{+}$

$$
\sum_{\substack{i_{1}, \ldots, i_{k} \in \mathbb{Z}_{+}, i_{1}+\cdots+i_{k} \leq j}} q^{-2(k-1) i_{1}-\cdots-2 i_{k}}=\frac{\left(q^{-2} ; q^{-2}\right)_{j+k}}{\left(q^{-2} ; q^{-2}\right)_{j}\left(q^{-2} ; q^{-2}\right)_{k}} .
$$

Denote the 1.h.s. of the previous equation by $\Psi(j, k)$. One can verify the recurrence relation for the $q$-Pascal triangle

$$
\Psi(j, k)=q^{-2 k} \Psi(j-1, k)+\Psi(j, k-1)
$$

and the boundary values

$$
\Psi(0, k)=1, \quad \Psi(j, 1)=\frac{1-q^{-2(j+1)}}{1-q^{-2}}
$$


explicitly. Thus $\Psi(j, k)$ are the corresponding entries of the $q$-Pascal triangle. Now we can complete our calculations and obtain that

$$
\begin{gathered}
\int_{\mathcal{H}_{n, m}} f\left(x_{n+1}\right) d \nu_{q}=\operatorname{const}_{1} \frac{q^{m(m-1)}}{\left(q^{2} ; q^{2}\right)_{m-1}} \sum_{i \in-\mathbb{Z}_{+}} f\left(q^{2 i}\right) q^{2 m i} \frac{\left(q^{-2} ; q^{-2}\right)_{n-1-i}}{\left(q^{-2} ; q^{-2}\right)_{-i}\left(q^{-2} ; q^{-2}\right)_{n-1}} \\
=\operatorname{const}_{1} \frac{q^{m(m-1)}}{\left(q^{2} ; q^{2}\right)_{m-1}} \sum_{i \in-\mathbb{Z}_{+}} f\left(q^{2 i}\right) q^{2 m i} \frac{\left(q^{2 i-2} ; q^{-2}\right)_{n-1}}{\left(q^{-2} ; q^{-2}\right)_{n-1}}=\int_{1}^{\infty} f(x) \rho(x) d_{q^{-2}} x
\end{gathered}
$$

where $\rho(x)=$ const $_{2} x^{m-1}\left(q^{-2} x-1\right)\left(q^{-4} x-1\right) \cdots\left(q^{-2(n-1)} x-1\right)$, and

$$
\text { const }_{2}=\frac{1}{q^{-2}-1} \text { const }_{1} \frac{q^{m(m-1)}}{\left(q^{2} ; q^{2}\right)_{m-1}} \frac{(-1)^{n-1}}{\left(q^{-2} ; q^{-2}\right)_{n-1}}=\frac{(-1)^{n-1}}{\left(q^{-2}-1\right)\left(q^{-2} ; q^{-2}\right)_{n-1}} .
$$

Now one can complete $\mathcal{D}\left(\mathcal{H}_{n, m}\right)_{q, \mathfrak{k}}^{U_{q}^{\mathfrak{k}}}$ with respect to the norm $\|f\|^{2}=\int_{1}^{\infty} f^{*} f \rho(x) d_{q^{-2}} x$. The resulting Hilbert space will be denoted by $L^{2}\left(d \nu_{q}^{(0)}\right)$.

\section{Covariant first order differential calculi over $\operatorname{Pol}\left(\widetilde{\mathcal{H}}_{n, m}\right)_{q}$}

In this section we introduce holomorphic and antiholomorphic covariant first order differential calculi over $\operatorname{Pol}\left(\widetilde{\mathcal{H}}_{n, m}\right)_{q}$.

First of all, recall a general definition of a covariant first order differential calculus following [8].

Let $F$ be a unital algebra. A first order differential calculus over $F$ is a pair $(M, d)$ where $M$ is an $F$-bimodule and $d: F \rightarrow M$ is a linear map such that

1. for all $f_{1}, f_{2} \in F$ one has $d\left(f_{1} \cdot f_{2}\right)=d f_{1} \cdot f_{2}+f_{1} \cdot d f_{2}$;

2. $M$ is a linear span of the vectors $f_{1} \cdot d f_{2} \cdot f_{3}$, where $f_{1}, f_{2}, f_{3} \in F$.

Now suppose that $A$ is a Hopf algebra and $F$ is an $A$-module algebra. A first order differential calculus $(M, d)$ over $F$ is called covariant if the following conditions hold:

1. $M$ is an $A$-covariant $F$-bimodule, i.e. the action maps $F \otimes M \rightarrow M, M \otimes F \rightarrow M$ are morphisms of $A$-modules;

2. $d$ is a morphism of $A$-modules.

Let $\mathcal{L}=L \circ \omega$, where $L$ is the canonical antirepresentation of $U_{q} \mathfrak{s l}_{N}$ in $\mathbb{C}\left[S L_{N}\right]_{q}$ (or the representation of $\left.U_{q} \mathfrak{s l}_{N}^{\text {op }}\right)$ and $\omega$ is an antiautomorphism of $U_{q} \mathfrak{s l}_{N}$ defined on the generators by $\omega\left(K_{i}\right)=K_{i}^{-1}, \omega\left(E_{i}\right)=F_{i}, \omega\left(F_{i}\right)=E_{i}$. Thus $\mathcal{L}$ is a representation of $U_{q} \mathfrak{s l}_{N}$ in $\mathbb{C}\left[S L_{N}\right]_{q}$.

Consider the action of $\mathcal{L}\left(E_{1} K_{1}^{-1 / 2}\right)$ in $\mathbb{C}\left[S L_{N}\right]_{q}$ and its restriction to $\operatorname{Pol}\left(\widetilde{\mathcal{H}}_{n, m}\right)_{q}$. Put

$$
\partial: \operatorname{Pol}\left(\widetilde{\mathcal{H}}_{n, m}\right)_{q} \rightarrow \operatorname{Pol}(\widetilde{X})_{q}, \quad \partial t_{i}=\mathcal{L}\left(E_{1} K_{1}^{-1 / 2}\right) t_{1 i}
$$

Let $\Omega^{(1,0)}\left(\widetilde{\mathcal{H}}_{n, m}\right)_{q} \subset \operatorname{Pol}(\widetilde{X})_{q}$ be the $\operatorname{Pol}\left(\widetilde{\mathcal{H}}_{n, m}\right)_{q}$-submodule generated by $\partial t_{i}, i=1, \ldots, N$.

Lemma 1. $\Omega^{(1,0)}\left(\widetilde{\mathcal{H}}_{n, m}\right)_{q}$ is a $U_{q} \mathfrak{s l}_{N}$-covariant first order differential calculus over $\operatorname{Pol}\left(\widetilde{\mathcal{H}}_{n, m}\right)_{q}$.

Proof. One has to verify the Leibniz rule which immediately follows from the formulas for the comultiplication in $U_{q} \mathfrak{s l}_{N}$. Since left and right action of $U_{q} \mathfrak{s l}_{N}$ in $\mathbb{C}\left[S L_{N}\right]_{q}$ commute, $\partial$ is a morphism of $U_{q} \mathfrak{s l}_{N}$-modules, so the calculus is covariant. 
Now we define $\bar{\partial}: \operatorname{Pol}\left(\widetilde{\mathcal{H}}_{n, m}\right)_{q} \rightarrow \operatorname{Pol}(\widetilde{X})_{q}$ by the rule $\bar{\partial} f=\left(\partial f^{*}\right)^{*}$. Let $\Omega^{(0,1)}\left(\widetilde{\mathcal{H}}_{n, m}\right)_{q} \subset$ $\operatorname{Pol}(\widetilde{X})_{q}$ be the $\operatorname{Pol}\left(\widetilde{\mathcal{H}}_{n, m}\right)_{q}$-submodule generated by $\bar{\partial} t_{i}, i=1, \ldots, N$.

Lemma 2. $\Omega^{(0,1)}\left(\widetilde{\mathcal{H}}_{n, m}\right)_{q}$ is a $U_{q} \mathfrak{s l}_{N}$-covariant first order differential calculus over $\operatorname{Pol}\left(\widetilde{\mathcal{H}}_{n, m}\right)_{q}$.

This lemma can be proved similarly to the previous one.

Remark 4. The introduced first order differential calculi can be obtained in another way. One should start from the canonical Wess-Zumino calculi on a quantum complex space introduced in [14] and then turn to a localization of the corresponding algebras of functions.

Now we introduce a Hermitian pairing $\Omega^{(0,1)}\left(\widetilde{\mathcal{H}}_{n, m}\right)_{q} \times \Omega^{(0,1)}\left(\widetilde{\mathcal{H}}_{n, m}\right)_{q} \rightarrow \operatorname{Pol}\left(\mathcal{H}_{n, m}\right)_{q}$.

Let $P: \operatorname{Pol}(\widetilde{X})_{q} \rightarrow \operatorname{Pol}\left(\mathcal{H}_{n, m}\right)_{q}$ be the projection parallel to a sum of other $U_{q} \mathfrak{s}\left(\mathfrak{g l}_{1} \times \mathfrak{g l} \mathfrak{g l}_{N-1}\right)$ isotypic components of $\mathcal{L}$. Now we define

$$
\left(\theta_{1}, \theta_{2}\right)=P\left(\theta_{2}^{*} \theta_{1}\right), \quad \theta_{1}, \theta_{2} \in \Omega^{(0,1)}\left(\widetilde{\mathcal{H}}_{n, m}\right)_{q} .
$$

By obvious commutativity of the left and right actions of $U_{q} \mathfrak{s l}_{N}$ we have

Proposition 5. The pairing $(\cdot, \cdot)$ is $U_{q} \mathfrak{s l}_{N}$-invariant.

\section{Lemma 3.}

$$
P\left(t_{2 j} t_{2 k}^{*}\right)=q^{-2} \frac{1-q^{2}}{1-q^{2(N-1)}}\left(\varepsilon_{j k}-t_{1 j} t_{1 k}^{*}\right),
$$

where

$$
\varepsilon_{j k}= \begin{cases}q^{2(j-1)}, & j=k, j \geq n+1, \\ -q^{2(j-1)}, & j=k, j \leq n, \\ 0, & j \neq k\end{cases}
$$

Proof. Since $U_{q} \mathfrak{s}\left(\mathfrak{g l}_{1} \times \mathfrak{g l}_{N-1}\right)=\mathbb{C}\left[K_{1}, K_{1}^{-1}\right] \otimes U_{q} \mathfrak{s l}_{N-1}$, one can decompose the projection $P$ as follows: $P=P_{0} P_{1}$, where $P_{1}$ is a projection to the subspace of $U_{q} \mathfrak{s l}_{N-1}$-invariant elements (w.r.t. the $\mathcal{L}$-action) and $P_{0}$ is a projection to the subspace of elements that are preserved by the $\mathcal{L}\left(K_{1}\right)$-action.

Let $u_{1}, \ldots, u_{k}$ be the standard basis in the $U_{q} \mathfrak{s l}_{k}$-module $L\left(\varpi_{1}\right)$, and $v_{1}, \ldots, v_{k}$ the dual basis in the $U_{q} \mathfrak{s l}_{k}$-module $L\left(\varpi_{k-1}\right)$, where $\varpi_{1}$ and $\varpi_{k-1}$ are the fundamental weights. A standard argument on finite dimensional $U_{q} \mathfrak{s l}_{k}$-modules allows one to prove that $\sum_{j=1}^{k}(-q)^{j-1} u_{j} \otimes v_{j}$ is a $U_{q} \mathfrak{s l}_{k}$-invariant element in the $U_{q} \mathfrak{s l}_{k}$-module $L\left(\varpi_{1}\right) \otimes L\left(\varpi_{k-1}\right)$, and the map

$$
u_{i} \otimes v_{j} \mapsto \begin{cases}\frac{1-q^{2}}{1-q^{2 k}}(-q)^{i-1} \sum_{a=1}^{k}(-q)^{a-1} u_{a} \otimes v_{a}, & i=j, \\ 0, & i \neq j,\end{cases}
$$

is a projection to the subspace of $U_{q} \mathfrak{s l}_{k}$-invariant elements parallel to other isotypic components. By obvious reasons, for $j=1, \ldots, N-1$ the maps

$$
\phi_{j}: u_{i} \mapsto t_{i+1, j}, \quad \psi_{j}: v_{i} \mapsto \operatorname{det}_{q} T_{i+1, j},
$$

admit extensions to morphisms of $U_{q} \mathfrak{s l}_{N-1}$-modules

$$
\phi_{j}: L\left(\varpi_{N-2}\right) \rightarrow \mathbb{C}\left[S L_{N}\right]_{q}, \quad \psi_{j}: L\left(\varpi_{1}\right) \rightarrow \mathbb{C}\left[S L_{N}\right]_{q}
$$


(w.r.t. the $\mathcal{L}$-action). By definition $(1), t_{i j}^{*}=(-q)^{j-i} \operatorname{det}_{q} T_{i j}$ for $i \leq m$ and $j \geq n+1$. Thus one can apply the map (10) to compute for $l \geq n+1$

$$
P_{1}\left(t_{2 l} t_{2 l}^{*}\right)=(-q)^{l-2} P_{1}\left(t_{2 l} \operatorname{det}_{q} T_{2 l}\right)=(-q)^{l-2} \frac{1-q^{2}}{1-q^{2(N-1)}} \sum_{j=1}^{N-1}(-q)^{j-1} t_{j+1, l} \operatorname{det}_{q} T_{j+1, l} .
$$

Since $\mathbb{C}\left[S L_{N}\right]_{q}$ is a Hopf algebra, one has $\sum_{j=1}^{N} t_{l j} S\left(t_{j} k\right)=\delta_{l k}$ and $S\left(t_{j k}\right)=(-q)^{j-k} \operatorname{det}_{q} T_{k j}$. Thus

$$
\begin{aligned}
P_{1}\left(t_{2 l} t_{2 l}^{*}\right) & =(-q)^{2(l-2)} \frac{1-q^{2}}{1-q^{2(N-1)}}\left(1-(-q)^{1-l} t_{1 l} \operatorname{det}_{q} T_{1 l}\right) \\
& =(-q)^{2(l-2)} \frac{1-q^{2}}{1-q^{2(N-1)}}\left(1-(-q)^{-2(l-1)} t_{1 l} t_{1 l}^{*}\right) .
\end{aligned}
$$

The other cases can be verified in a similar way.

\section{Proposition 6.}

$$
\left(\bar{\partial} x_{n+1}, \bar{\partial} x_{n+1}\right)=q^{2(n-1)} \frac{1-q^{2}}{1-q^{2(N-1)}} x_{n+1}\left(1-q^{-2 n} x_{n+1}\right) .
$$

Proof. An easy application of the previous lemma allows one to compute that

$$
\left(\bar{\partial}\left(t_{1 k}^{*} t_{1 l}\right), \bar{\partial}\left(t_{1 j}^{*} t_{1 i}\right)\right)=q^{-2} \frac{1-q^{2}}{1-q^{2(N-1)}}\left(\varepsilon_{j k} t_{1 i}^{*} t_{1 l}-\left(t_{1 i}^{*} t_{1 j}\right)\left(t_{1 k}^{*} t_{1 l}\right)\right) .
$$

Thus for $j, k \geq n+1$ one has

$$
\begin{aligned}
& \left(\bar{\partial}\left(q^{-2 j} t_{1 j}^{*} t_{1 j}\right), \bar{\partial}\left(q^{-2 k} t_{1 k}^{*} t_{1 k}\right)\right) \\
& \quad=q^{-2} \frac{1-q^{2}}{1-q^{2(N-1)}}\left(\delta_{j k} q^{-2(j+1)} t_{1 j}^{*} t_{1 j}-\left(q^{-2 j} t_{1 j}^{*} t_{1 j}\right)\left(q^{-2 k} t_{1 k}^{*} t_{1 k}\right)\right) .
\end{aligned}
$$

By easy computations, $q^{-2(n+1)} x_{n+1}=\sum_{j=n+1}^{N} q^{-2 j} t_{1 j}^{*} t_{1 j}$, which enables to prove the claim.

Let us fix notation for $q$-difference operators:

$$
B_{-}: f(x) \mapsto \frac{f\left(q^{-2} x\right)-f(x)}{q^{-2} x-x}, \quad B_{+}: f(x) \mapsto \frac{f\left(q^{2} x\right)-f(x)}{q^{2} x-x} .
$$

\section{Lemma 4.}

$$
\left(\bar{\partial} f\left(x_{n+1}\right), \bar{\partial} g\left(x_{n+1}\right)\right)=q^{2(n-1)} \frac{1-q^{2}}{1-q^{2(N-1)}} x_{n+1}\left(1-q^{-2 n} x_{n+1}\right) \overline{B_{-} g\left(x_{n+1}\right)} B_{-} f\left(x_{n+1}\right) .
$$

Proof. By explicit calculations in $\mathbb{C}\left[S L_{N}\right]_{q}$ we obtain for $i \geq n+1$

$$
t_{1 i} \bar{\partial} x_{n+1}=q^{-1} \bar{\partial} x_{n+1} t_{1 i}, \quad t_{1 i}^{*} \bar{\partial} x_{n+1}=q^{-1} \bar{\partial} x_{n+1} t_{1 i}^{*} .
$$

Let us verify the second identity. One has $\bar{\partial} x_{n+1}=\sum_{j=n+1}^{N} t_{1 j} t_{2 j}^{*}$, so

$$
t_{1 i}^{*} \bar{\partial} x_{n+1}=t_{1 i}^{*}\left(\sum_{j=n+1}^{i-1} t_{1 j} t_{2 j}^{*}+t_{1 i} t_{2 i}^{*}+\sum_{j=i+1}^{N} t_{1 j} t_{2 j}^{*}\right)=q^{-1} \sum_{j=n+1}^{i-1} t_{1 j} t_{2 j}^{*} t_{1 i}^{*}
$$




$$
+q^{-1} t_{1 i} t_{2 i}^{*} t_{1 i}^{*}+\left(q-q^{-1}\right) \sum_{j>i} t_{1 j} t_{1 j}^{*} t_{2 i}^{*}+q^{-1} \sum_{j=i+1}^{N} t_{1 j} t_{2 j}^{*} t_{1 i}^{*}+\left(q^{-1}-q\right) \sum_{j>i} t_{1 j} t_{1 j}^{*} t_{2 i}^{*} .
$$

So we have $x_{n+1} \bar{\partial} x_{n+1}=q^{-2} \bar{\partial} x_{n+1} x_{n+1}$. Hence for every polynomial $f(x)$

$$
\bar{\partial} f\left(x_{n+1}\right)=\bar{\partial} x_{n+1} \frac{f\left(q^{-2} x_{n+1}\right)-f\left(x_{n+1}\right)}{q^{-2} x_{n+1}-x_{n+1}}=\bar{\partial} x_{n+1} B_{-}(f)\left(x_{n+1}\right),
$$

and the claim follows from the previous proposition.

Using the above formal arguments, we can extend $\bar{\partial}$ to $\mathcal{D}\left(\mathcal{H}_{n, m}\right)_{q, \mathfrak{k}}$.

\section{The Laplace-Beltrami operator and its radial part}

In this section we introduce a $U_{q} \mathfrak{s l}_{N}$-invariant operator $\square$ in the space of finite functions $\mathcal{D}\left(\mathcal{H}_{n, m}\right)_{q, \mathfrak{k}}$. This operator will be considered as a quantum analog for the invariant LaplaceBeltrami operator on the complex hyperbolic space $\mathcal{H}_{n, m}$. Also we compute an explicit formula for the restriction $\square^{(0)}$ of $\square$ to the space $\mathcal{D}\left(\mathcal{H}_{n, m}\right)_{q, \mathfrak{k}}^{U_{q}^{\mathfrak{k}}}$, the so called radial part of $\square$. The restriction appears to be a $q$-difference operator in variable $x=x_{n+1}$.

Now we define $\square$ by the formula

$$
\int_{\mathcal{H}_{n, m}} f_{2}^{*}\left(\square f_{1}\right) d \nu_{q}=\int_{\mathcal{H}_{n, m}}\left(\bar{\partial} f_{1}, \bar{\partial} f_{2}\right) d \nu_{q}, \quad f_{1}, f_{2} \in \mathcal{D}\left(\mathcal{H}_{n, m}\right)_{q, \mathfrak{k}} .
$$

Proposition 7. $\square$ is a self-adjoint $U_{q} \mathfrak{s l}_{N}$-invariant operator.

Proof. The self-adjointness follows from the definition. The $U_{q} \mathfrak{s l}_{N}$-invariance of the form $(\cdot, \cdot)$ and the linear functional $\int_{\mathcal{H}_{n, m}} \cdot d \nu_{q}$ implies the $U_{q} \mathfrak{s l}_{N}$-invariance of $\square$.

Let us find the restriction $\square^{(0)}$ of the operator $\square$ on the subspace

$$
\mathcal{D}\left(\mathcal{H}_{n, m}\right)_{q, \mathfrak{k}}^{U_{q}^{\mathfrak{k}}}=\left\{f\left(x_{n+1}\right), \operatorname{supp} f \subset q^{-2 \mathbb{Z}_{+}}, \sharp(\operatorname{supp} f)<\infty\right\},
$$

based on the equation

$$
\begin{aligned}
\int_{1}^{\infty} & \left(\square^{(0)} f\right)(x) \overline{f(x)} \rho(x) d_{q^{-2}} x \\
& =q^{2(n-1)} \frac{1-q^{2}}{1-q^{2(N-1)}} \int_{1}^{\infty} x\left(1-q^{-2 n} x\right)\left|\frac{f\left(q^{-2} x\right)-f(x)}{q^{-2} x-x}\right|^{2} \rho(x) d_{q^{-2}} x
\end{aligned}
$$

for every $f$ with finite support.

Using the $q$-analog of the partial integration formulas one can prove that operators $B_{-}$and $-q^{2} B_{+}$are formally dual. Exactly, for every functions $u(x), v(x)$ with finite support on $q^{2 \mathbb{Z}}$ holds

$$
\int_{0}^{\infty} u(x) \frac{v\left(q^{-2} x\right)-v(x)}{\left(q^{-2}-1\right) x} d_{q^{-2}} x=-q^{2} \int_{0}^{\infty} \frac{u(x)-u\left(q^{2} x\right)}{\left(1-q^{2}\right) x} v(x) d_{q^{-2}} x,
$$

where

$$
\int_{0}^{\infty} f(x) d_{q^{-2}} x=\left(q^{-2}-1\right) \sum_{k=-\infty}^{\infty} f\left(q^{-2 k}\right) q^{-2 k}
$$


Thus

$$
\square^{(0)}: \quad f(x) \mapsto \operatorname{const}(q, n, N) \rho(x)^{-1} B_{+} x\left(q^{-2 n} x-1\right) \rho(x) B_{-} f(x),
$$

where

$$
\operatorname{const}(q, n, N)=q^{2 n} \frac{1-q^{2}}{1-q^{2(N-1)}},
$$

and $\rho(x)$ is defined in (9).

Lemma 4 allows us to extend the Hermitian $U_{q} \mathfrak{s l}_{N}$-invariant pairing to first order differential forms with coefficients in $\mathcal{D}\left(\mathcal{H}_{n, m}\right)_{q, \mathfrak{k}}$.

Lemma 5. For every function $f(x)$ with finite support on $q^{-2 \mathbb{Z}_{+}}$

$$
\begin{aligned}
\square^{(0)} f(x)= & \frac{q^{2}}{\left(1-q^{2}\right)^{2} x}\left((x-1) q^{2 m-2} f\left(q^{2} x\right)+\left(q^{-2 n} x-1\right) f\left(q^{-2} x\right)\right. \\
& \left.+\left(1+q^{2 m-2}-q^{2 m-2} x-q^{-2 n} x\right) f(x)\right) .
\end{aligned}
$$

Proposition 8. The operator $\square^{(0)}$ is bounded.

Proof. Consider the operator

$$
\tilde{\square}: f(x) \mapsto x^{-(N-1)} \operatorname{const}(q, n, N) q^{-2 n} B_{+} x^{N+1} B_{-} f(x) .
$$

It differs from $\square^{(0)}$ by a compact operator, so it is sufficient to prove that $\widetilde{\square}$ is bounded. The boundness of the latter operator can be proved by the direct evaluation.

By the previous proposition, one can extend $\square^{(0)}$ from $\mathcal{D}\left(\mathcal{H}_{n, m}\right)_{q, \mathfrak{k}}^{U_{q}^{\mathfrak{k}}}$ to a bounded self-adjoint operator in $L^{2}\left(d \nu_{q}^{(0)}\right)$. The latter extension will also be denoted by $\square^{(0)}$.

\section{Generalized eigenfunctions of $\square^{(0)}$ and Al-Salam-Chihara polynomials}

In this section we obtain the initial results on the bounded self-adjoint operator $\square^{(0)}$, namely we obtain its formal eigenfunctions and eigenvalues explicitly. Note that explicit computations of the asymptotics of these eigenfunctions, as in the classical case, allow us to consider a quantum analog of the Harish-Chandra $c$-function (see Appendix A).

By the direct computation we obtain the following lemma.

Lemma 6. The function

$$
\Phi_{l}(x)={ }_{3} \Phi_{2}\left(\begin{array}{c}
x, q^{-2 l}, q^{2(l+N-1)} \\
q^{2 n}, 0
\end{array} q^{2}, q^{2}\right)
$$

in $\mathcal{D}\left(\mathcal{H}_{n, m}\right)_{q}^{\prime}$ is a generalized eigenfunction for $\square^{(0)}$ :

$$
\square^{(0)} \Phi_{l}=\lambda(l) \Phi_{l}
$$

with the eigenvalue

$$
\lambda(l)=-q^{2-2 n} \frac{\left(1-q^{-2 l}\right)\left(1-q^{2 l+2(N-1)}\right)}{\left(1-q^{2}\right)^{2}} .
$$


Recall the definition of the Al-Salam-Chihara polynomials, following [9]:

$$
Q_{k}(z ; a, b \mid q)=\frac{(a b ; q)_{k}}{a^{k}}{ }_{3} \Phi_{2}\left(\begin{array}{c}
q^{-k}, a e^{i \theta}, a e^{-i \theta} \\
a b, 0
\end{array} ; q, q\right), \quad z=\cos \theta
$$

We have the following well known orthogonality relations for the Al-Salam-Chihara polynomials:

$$
\begin{aligned}
& \frac{1}{2 \pi} \int_{-1}^{1} Q_{i}(z ; a, b \mid q) Q_{j}(z ; a, b \mid q) \frac{w(z)}{\sqrt{1-z^{2}}} d z \\
& \quad+\sum_{1<a q^{k}<a} w_{k} Q_{i}\left(z_{k} ; a, b \mid q\right) Q_{j}\left(z_{k} ; a, b \mid q\right)=\frac{\delta_{i j}}{\left(q^{i+1}, a b q^{i} ; q\right)_{\infty}}
\end{aligned}
$$

where

$$
\begin{gathered}
w(z)=\frac{h(z, 1) h(z,-1) h\left(z, q^{1 / 2}\right) h\left(z,-q^{1 / 2}\right)}{h(z, a) h(z, b)}, \quad h(z, a)=\left(a e^{i \theta}, a e^{-i \theta} ; q\right)_{\infty}, \quad z=\cos \theta, \\
z_{k}=\frac{a q^{k}+a q^{-k}}{2} \text {, and } \\
w_{k}=\frac{\left(a^{-2} ; q\right)_{\infty}}{(q, a b, b / a ; q)_{\infty}} \frac{\left(1-a^{2} q^{2 k}\right)\left(a^{2}, a b ; q\right)_{k}}{\left(1-a^{2}\right)(q, q a / b ; q)_{k}} q^{-k^{2}}\left(\frac{1}{a^{3} b}\right)^{k} .
\end{gathered}
$$

Also, these polynomials satisfy the following recurrence relations:

$$
\begin{aligned}
z Q_{i}(z ; a, b \mid q)= & \frac{1}{2} Q_{i+1}(z ; a, b \mid q)+\frac{1}{2} q^{i}(a+b) Q_{i}(z ; a, b \mid q) \\
& +\frac{1}{2}\left(1-q^{i}\right)\left(1-a b q^{i-1}\right) Q_{i-1}(z ; a, b \mid q)
\end{aligned}
$$

As one can see, the eigenfunctions $\Phi_{l}(x)$ are connected with the Al-Salam-Chihara polynomials:

$$
\Phi_{l}\left(q^{-2 k}\right)=\frac{q^{k(N-1)}}{\left(q^{2 n} ; q^{2}\right)_{k}} Q_{k}\left(z ; q^{2 n-N+1}, q^{N-1} \mid q^{2}\right), \quad e^{i \theta}=q^{2 l+N-1}, \quad z=\cos \theta .
$$

Let us denote the orthogonal measure for Al-Salam-Chihara polynomials by $d \sigma$. Note that if $2 n-N+1>0$, the sum in the orthogonality relations vanishes and we have just the continuous measure.

\section{A spectral theorem for the radial part of}

In this section we obtain a spectral theorem for $\square^{(0)}$. As in the classical case [5, pp. 429-432], the support of the Plancherel measure consists of continuous and discrete parts. The continuous part corresponds to principal unitary series of $U_{q} \mathfrak{s u}{ }_{n, m}$-modules related to a quantum analog of the cone

$$
\Xi_{n, m}=\left\{x \in \mathbb{C}^{N} \mid-x_{1} \bar{x}_{1}-\cdots-x_{n} \bar{x}_{n}+x_{n+1} \bar{x}_{n+1}+\cdots+x_{N} \bar{x}_{N}=0\right\}
$$

(this series is established in [3]). The discrete part is supposed to be corresponded to a discrete series of unitary $U_{q} \mathfrak{s u} \mathfrak{u}_{n, m}$-modules. 
Theorem 3. The bounded self-adjoint linear operator $\square^{(0)}$ is unitary equivalent to the operator of multiplication by independent variable in the Hilbert space $L^{2}(d \sigma)$. The unitary equivalence is given by the operator

$$
\begin{aligned}
& U: L^{2}\left(d \nu_{q}^{(0)}\right) \rightarrow L^{2}(d \sigma) \\
& U: f(x) \mapsto \hat{f}(\lambda)=\int_{1}^{\infty} f(x) \Phi_{l}(x) \rho(x) d_{q^{-2}} x,
\end{aligned}
$$

where $\lambda=-q^{2-2 n} \frac{\left(1-q^{-2 l}\right)\left(1-q^{2 l+2 N-2}\right)}{\left(1-q^{2}\right)^{2}}$.

Proof. Let us consider the finite functions on $q^{-2 \mathbb{Z}_{+}}$

$$
f_{j}(x)=\left\{\begin{array}{ll}
1, & x=q^{-2 j}, \\
0, & \text { otherwise },
\end{array} \quad j \in \mathbb{Z}_{+} .\right.
$$

These functions form an orthogonal system in $\mathcal{D}\left(\mathcal{H}_{n, m}\right)_{q, \mathfrak{k}}^{U_{q} \mathfrak{k}}$ and the completion of its linear span is $L^{2}\left(d \nu_{q}^{(0)}\right)$. By standard arguments [1], the bounded self-adjoint linear operator $\square^{(0)}$ is unitary equivalent to the multiplication operator $f(\lambda) \mapsto \lambda f(\lambda)$ in the Hilbert space $L^{2}(d \mu(\lambda))$ of square integrable functions with respect to a certain measure $d \mu(\lambda)$ with compact support in $\mathbb{R}$. Let us find explicitly the corresponding measure and the operator of unitary equivalence $U$. One can fix the unitary equivalence operator by the condition $U f_{0}=1$.

By easy calculations, $\square^{(0)}$ transforms $f_{j}$ by the formula

$$
\begin{aligned}
\square^{(0)} f_{j}= & \frac{q^{2}}{\left(1-q^{2}\right)^{2}}\left(f_{j+1}\left(1-q^{2 j+2}\right) q^{2 m-2}+f_{j-1}\left(1-q^{2 j+2 n-2}\right) q^{-2 n}\right. \\
& \left.+f_{j}\left(q^{2 j}+q^{2 j+2 m-2}-q^{2 m-2}-q^{-2 n}\right)\right), \quad j \in \mathbb{Z}_{+},
\end{aligned}
$$

and $\left\|f_{j}\right\|^{2}=q^{-2 j(N-1)} \frac{\left(q^{2 j+2} ; q^{2}\right)_{n-1}}{\left(q^{2} ; q^{2}\right)_{n-1}}$ (here we naturally suppose $f_{-1}=0$ ).

Thus the finite functions $e_{j}=q^{j(N-1)} \sqrt{\frac{\left(q^{2} ; q^{2}\right)_{n-1}}{\left(q^{2 j+2} ; q^{2}\right)_{n-1}}} f_{j}$ form an orthonormal system in $L^{2}\left(d \nu_{q}^{(0)}\right)$ and $\square^{(0)}$ acts on them by the formula

$$
\begin{aligned}
\square^{(0)} e_{j}= & \frac{q^{m-n+1}}{\left(1-q^{2}\right)^{2}}\left(e_{j+1} \sqrt{\left(1-q^{2 j+2}\right)\left(1-q^{2 j+2 n}\right)}+e_{j-1} \sqrt{\left(1-q^{2 j}\right)\left(1-q^{2 j+2 n-2}\right)}\right. \\
& \left.+e_{j}\left(q^{2 j+(N-1)}+q^{2 j+2 n-(N-1)}-q^{N-1}-q^{1-N}\right)\right), \quad j \in \mathbb{Z}_{+} .
\end{aligned}
$$

Thus $P_{j}=U e_{j} \in L^{2}(d \mu(\lambda)), j \in \mathbb{Z}_{+}$form an orthonormal system of polynomials

$$
\int P_{i}(\lambda) P_{j}(\lambda) d \mu(\lambda)=\delta_{i j}, \quad i, j \in \mathbb{Z}_{+}
$$

and one has

$$
\begin{aligned}
\lambda P_{j}(\lambda)= & \frac{q^{m-n+1}}{\left(1-q^{2}\right)^{2}}\left(P_{j+1}(\lambda) \sqrt{\left(1-q^{2 j+2}\right)\left(1-q^{2 j+2 n}\right)}\right. \\
& +P_{j-1}(\lambda) \sqrt{\left(1-q^{2 j}\right)\left(1-q^{2 j+2 n-2}\right)} \\
& \left.+P_{j}(\lambda)\left(q^{2 j+(N-1)}+q^{2 j+2 n-(N-1)}-q^{N-1}-q^{1-N}\right)\right), \quad j \in \mathbb{Z}_{+}, \\
P_{0}(\lambda)= & U e_{0}=U f_{0}=1 .
\end{aligned}
$$

(we naturally suppose that $P_{-1}=0$ ). 
The orthogonal polynomials $P_{j}(\lambda), j \in \mathbb{Z}_{+}$are determined by (13) and (14). Let us compare them with the corresponding recurrence relations (12) and the initial data for the Al-SalamChihara polynomials $Q_{j}\left(z ; q^{2 n-(N-1)}, q^{N-1} \mid q^{2}\right)$. One can observe that

$$
\left\|Q_{j}\right\|^{2}=\frac{1}{\left(q^{2 j+2}, q^{2 j+2 n} ; q^{2}\right)_{\infty}}=\frac{\left(q^{2}, q^{2 n} ; q^{2}\right)_{j}}{\left(q^{2} ; q^{2}\right)_{n-1}\left(q^{2 n} ; q^{2}\right)_{\infty}^{2}} .
$$

The polynomials

$$
\widetilde{Q}_{j} \stackrel{\text { def }}{=} \sqrt{\frac{1}{\left(q^{2}, q^{2 n} ; q^{2}\right)_{j}}} Q_{j}
$$

satisfy the following recurrence relations:

$$
\begin{aligned}
z \widetilde{Q}_{j}= & \frac{1}{2} \sqrt{\left(1-q^{2(j+1)}\right)\left(1-q^{2 j+2 n}\right)} \widetilde{Q}_{j+1}+\frac{1}{2} \sqrt{\left(1-q^{2 j}\right)\left(1-q^{2 j+2 n-2}\right)} \widetilde{Q}_{j-1} \\
& +\frac{1}{2} q^{2 j}\left(q^{2 n-N+1}+q^{N-1}\right) \widetilde{Q}_{j}, \quad j \in \mathbb{Z}_{+} .
\end{aligned}
$$

Thus we obtain that $P_{j}$ and $\widetilde{Q}_{j}$ are related by the change of variable

$$
\lambda=\frac{2 q^{m-n+1}}{\left(1-q^{2}\right)^{2}} z-\frac{q^{m-n+1}}{\left(1-q^{2}\right)^{2}}\left(q^{N-1}+q^{-(N-1)}\right) .
$$

So,

$$
P_{j}(\lambda)=\sqrt{\frac{1}{\left(q^{2}, q^{2 n} ; q^{2}\right)_{j}}} Q_{j}\left(z ; q^{2 n-N+1}, q^{N-1} \mid q^{2}\right),
$$

where $z=\cos \theta=\frac{e^{i \theta}+e^{-i \theta}}{2}$ and

$$
\begin{aligned}
\lambda= & -\frac{q^{2-2 n}}{\left(1-q^{2}\right)^{2}}\left(1-q^{N-1} e^{i \theta}\right)\left(1-q^{N-1} e^{-i \theta}\right), \\
U f_{j} & =q^{-j(N-1)} \sqrt{\frac{\left(q^{2 j+2} ; q^{2}\right)_{n-1}}{\left(q^{2} ; q^{2}\right)_{n-1}}} \sqrt{\frac{1}{\left(q^{2}, q^{2 n} ; q^{2}\right)_{j}}} Q_{j}\left(z ; q^{2 n-N+1}, q^{N-1} \mid q^{2}\right) \\
& =q^{-j(N-1)} \frac{1}{\left(q^{2} ; q^{2}\right)_{j}} Q_{j}\left(z ; q^{2 n-N+1}, q^{N-1} \mid q^{2}\right) .
\end{aligned}
$$

On the other hand,

$$
\int_{1}^{\infty} f_{j}(x) \Phi_{l}(x) \rho(x) d_{q^{-2}} x=\frac{q^{-j(N-1)}}{\left(q^{2} ; q^{2}\right)_{j}} Q_{j}\left(z ; q^{2 n-N+1}, q^{N-1} \mid q^{2}\right),
$$

where $z=\frac{1}{2}\left(q^{2 l+N-1}+q^{-(2 l+N-1)}\right)$.

Hence for every function $f(x)$ on $q^{-2 \mathbb{Z}_{+}}$with finite support one has

$$
U f=\int_{1}^{\infty} f(x) \Phi_{l}(x) \rho(x) d_{q^{-2}} x .
$$

Now the claim of the theorem follows from the orthogonality relations for the Al-Salam-Chihara polynomials (11). 
Remark 5. There is an extensive literature on harmonic analysis related to a quantum $S U_{q}(1,1)$ (see the references $[10,18,20]$ from three research groups and references therein). Even the notion of quantum $S U_{q}(1,1)$ had some uncertainties back then (unlike to the case of quantum compact group $\left.S U_{q}(2)\right)$. A remarkable quantum effect lies in the fact that the Plancherel type theorems for the quantum $S U_{q}(1,1)$ present decompositions with entries coming from the principal unitary series and strange unitary series of representations (which also has no classical analog).

Later on L. Vaksman established a new concept of quantum $S U_{q}(1,1)$ (and other noncompact real Lie groups). He substituted the group $S U(1,1) \subset S L_{2}(\mathbb{C})$ by its principal homogeneous

space $w_{0} S U(1,1)$, where $w_{0}=\left(\begin{array}{cc}0, & -1 \\ 1, & 0\end{array}\right)$. This enables him and his collaborators to prove the Plancherel type theorems with decompositions that does not contain the strange series entries $($ see $[16,2])$. Still a relation between these two approaches of quantization may require further investigations.

\section{A Appendix on the Harish-Chandra $c$-function and the Plancherel measure}

Let us introduce the notation of the $q$-analog of the Harish-Chandra $c$-function

$$
c(l)=\frac{\Gamma_{q^{2}}(n) \Gamma_{q^{2}}(2 l+N-1)}{\Gamma_{q^{2}}(l+n) \Gamma_{q^{2}}(l+N-1)}=\frac{\left(q^{2(l+N-1)} ; q^{2}\right)_{\infty}\left(q^{2(l+n)} ; q^{2}\right)_{\infty}}{\left(q^{2(2 l+N-1)} ; q^{2}\right)_{\infty}\left(q^{2 n} ; q^{2}\right)_{\infty}},
$$

where $\Gamma_{q}(x)=(1-q)^{1-x} \frac{(q ; q)_{\infty}}{\left(q^{x} ; q\right)_{\infty}}$ is a well known quantum analog for the Gamma-function [6].

\section{Lemma 7.}

1. For $\operatorname{Re} l<-(N-1) / 2$ one has $\Phi_{l}(x) \sim c(-l-N+1) x^{-l-N+1}$ as $x \in q^{-2 \mathbb{Z}_{+}}$tends to infinity.

2. For $\operatorname{Re} l>-(N-1) / 2$ one has $\Phi_{l}(x) \sim c(l) x^{l}$ as $x \in q^{-2 \mathbb{Z}_{+}}$tends to infinity.

Proof. Let us compute an asymptotic behavior of $\Phi_{l}(x)$ for $\operatorname{Re} l>-(N-1) / 2$, the other case can be managed similarly. For $x=q^{-2 k}, k \rightarrow \infty$, one has

$$
\begin{aligned}
& { }_{3} \Phi_{2}\left(\begin{array}{c}
q^{-2 k}, q^{-2 l}, q^{2(l+N-1)} \\
q^{2 n}, 0
\end{array} q^{2}, q^{2}\right)=\frac{\left(q^{2-2 l-2 n-2 k} ; q^{2}\right)_{k}}{\left(q^{2-2 n-2 k} ; q^{2}\right)_{k}} \Phi_{1}\left(\begin{array}{c}
q^{-2 k}, q^{-2 l} \\
q^{2-2 l-2 n-2 k}
\end{array} ; q^{2}, q^{2 l+2 m}\right) \\
& \sim q^{-2 l k} \frac{\left(q^{2 l+2 n} ; q^{2}\right)_{\infty}}{\left(q^{2 n} ; q^{2}\right)_{\infty}} \Phi_{0}\left(\begin{array}{c}
q^{-2 l} \\
-
\end{array} q^{2}, q^{4 l+2(N-1)}\right) \\
& =q^{-2 l k} \frac{\left(q^{2 l+2 n} ; q^{2}\right)_{\infty}\left(q^{2 l+2(N-1)} ; q^{2}\right)_{\infty}}{\left(q^{2 n} ; q^{2}\right)_{\infty}\left(q^{4 l+2(N-1)} ; q^{2}\right)_{\infty}} .
\end{aligned}
$$

Let us return to the Plancherel measure of $\square^{(0)}$, namely for its absolutely continuous part. Since $e^{i \theta}=q^{2 l+N-1}$ and $z=\cos \theta$, one can observe by easy calculations that

$$
\begin{aligned}
w(z)=w\left(z, q^{2 n-N+1}, q^{N-1} \mid q^{2}\right) & =\frac{\left(q^{4 l+2(N-1)}, q^{-4 l-2(N-1)} ; q^{2}\right)_{\infty}}{\left(q^{2 l+2 n}, q^{2 n-2 l-2(N-1)}, q^{2 l+2(N-1)}, q^{-2 l} ; q^{2}\right)_{\infty}} \\
& =\frac{1}{c(l) c(-l-(N-1))} \frac{1}{\left(q^{2 n} ; q^{2}\right)_{\infty}^{2}} .
\end{aligned}
$$

By this calculations we establish a relation between continuous part of the Plancherel measure and the Harish-Chandra $c$-function in the quantum case. Of course, this interplay in the classical $(q=1)$ situation is well-known. 


\section{Acknowledgements}

This project started out as joint work with L. Vaksman and D. Shklyarov. We are grateful to both of them for helpful discussions and drafts with preliminary definitions and computations. Also we are grateful for referees for their comments that help to improve and simplify our exposition.

\section{References}

[1] Akhiezer N.I., Glazman I.M., Theory of linear operators in Hilbert space, Dover Publications, New York, 1993.

[2] Bershtein O., Kolisnyk Ye., Plancherel measure for the quantum matrix ball. I, J. Math. Phys. Anal. Geom. 5 (2009), 315-346, arXiv:0903.4068.

[3] Bershtein O., Sinelshchikov S., Function theory on a $q$-analog of complex hyperbolic space, arXiv:1009.6063.

[4] Chari V., Pressley A., A guide to quantum groups, Cambridge University Press, Cambridge, 1994.

[5] Faraut J., Distributions sphériques sur les espaces hyperboliques, J. Math. Pures Appl. 58 (1979), 369-444.

[6] Gaspar G., Rahman M., Basic hypergeometric series, Encyclopedia of Mathematics and its Applications, Vol. 35, Cambridge University Press, Cambridge, 1990.

[7] Jantzen J.C., Lectures on quantum groups, Graduate Studies in Mathematics, Vol. 6, American Mathematical Society, Providence, RI, 1996.

[8] Klimyk A., Schmüdgen K., Quantum groups and their representations, Texts and Monographs in Physics, Springer-Verlag, Berlin, 1997.

[9] Koekoek R., Swarttouw R.F., The Askey-scheme of hypergeometric orthogonal polynomials and its $q$ analogue, Report 98-17, Faculty of Technical Mathematics and Informatics, Delft University of Technology, 1998, http://aw.twi.tudelft.nl/ koekoek/askey/.

[10] Koelink E., Stokman J.V., Fourier transforms on the quantum $S U(1,1)$ group, with an appendix by M. Rahman, Publ. Res. Inst. Math. Sci. 37 (2001), 621-715, math.QA/9911163.

[11] Molchanov V.F., Spherical functions on hyperboloids, Mat. Sb. 99 (1976), no. 2, 139-161 (English transl.: Math. USSR Sb. 28 (1976), no. 2, 119-139).

[12] Molchanov V.F., Harmonic analysis on homogeneous spaces, Itogi Nauki i Tekhniki, Vol. 59, VINITI, Moscow, 1990, 5-144 (English transl.: Encycl. Math. Sci., Vol. 59, Springer, Berlin, 1995, 1-135).

[13] Onishchik A.L., Vinberg E.B., Lie groups and algebraic groups, Springer Series in Soviet Mathematics, Springer-Verlag, Berlin, 1990.

[14] Reshetikhin N.Yu., Takhtadzhyan L.A., Faddeev L.D., Quantization of Lie groups and Lie algebras, Algebra i Analiz 1 (1989), 178-206 (English transl.: Leningrad Math. J. 1 (1990), 193-225).

[15] Shklyarov D., Sinel'shchikov S., Vaksman L., Fock representations and quantum matrices, Internat. J. Math. 15 (2004), 855-894, math.QA/0410605.

[16] Shklyarov D., Sinel'shchikov S., Vaksman L., On function theory on quantum disc: $q$-differential equations and Fourier transform, math.QA/9809002.

[17] Shklyarov D., Sinel'shchikov S., Stolin A., Vaksman L., On a $q$-analogue of the Penrose transform, Ukr. Phys. J. 47 (2003), 288-292.

[18] Ueno K., Spectral analysis for the Casimir operator on the quantum group SU(1,1), Proc. Japan Acad. Ser. A Math. Sci. 66 (1990), no. 2, 42-44.

[19] Vaksman L.L., Quantum bounded symmetric domains, translated by O. Bershtein and S. Sinel'shchikov, Translations of Mathematical Monographs, Vol. 238, American Mathematical Society, Providence, RI, 2010.

[20] Vaksman L.L., Korogodskii L.I., Spherical functions on the quantum group SU(1,1) and a $q$-analogue of the Mehler-Fock formula, Funktsional. Anal. i Prilozhen. 25 (1991), no. 1, 60-62 (English transl.: Funct. Anal. Appl. 25 (1991), no. 1, 48-49).

[21] van Dijk G., Sharshov Yu.A., The Plancherel formula for line bundles on complex hyperbolic spaces, J. Math. Pures Appl. 79 (2000), 451-473. 